\title{
Joint acquisition algorithm with assisted information for weak GNSS signals
}

\author{
Haibo Tong*, Hang Gong, Guozhu Zhang and Gang Ou
}

\begin{abstract}
In this paper, we propose an enhanced joint acquisition scheme with satellite selection and assisted information to detect weak signals. The theoretical derivation of the joint acquisition is detailed in the principle of the generalized likelihood ratio test (GLRT) to evaluate detection performance. An approximate and simplified architecture is proposed to reduce the computation load. The traditional acquisition performs two-dimensional search over the delay and Doppler frequency per satellite, while the simplified joint acquisition adopts one statistic for all the available satellites to perform multi-dimensional search over the receiver motion parameters and the clock errors. Based on the simplified architecture, one novel strategy of the satellite selection is presented to enhance the robustness. The selection strategy removes the satellites which should be available but are possibly blocked by buildings or trees. Monte Carlo simulations are performed to verify the analytical results. Under the condition that 1-ms coherent integration is adopted in the simulation with 16 satellites, the sensitivity improvement of the enhanced joint acquisition is about $9 \mathrm{~dB}$ over those of conventional acquisition. Furthermore, experiments show that the joint acquisition with the satellite selection is more invulnerable to blocked satellites than that without the satellite selection.
\end{abstract}

Keywords: Assisted GNSS; Acquisition; Weak signals; Satellite selection; Generalized likelihood ratio test

\section{Introduction}

As new constellations become operational, global navigation satellite systems (GNSS) are expected to provide better positioning service in the fading environments (e.g., indoors, urban canyon, and flyover). Long integration time is always used in the conventional scalar-based acquisition and tracking for weak signals $[1,2]$. Various methods on the coherent, noncoherent, and differential detectors were analyzed theoretically in [3-6]. However, the sensitivity improvement of the conventional acquisition is limited by the constraints, such as receiver movement and computational load. Due to each satellite signal that is processed individually, the correlations of the multi-satellite signals are neglected by the scalarbased acquisition and tracking. If the correlations of the GNSS signals from different satellites are exploited, the acquisition sensitivity will be improved further.

Instead of processing the signals separately, the vector delay/frequency lock loop (VDFLL) adopts a single

*Correspondence: hbo.tong@gmail.com

College of Electronic Science and Engineering, National University of Defense Technology, Deya Road 109, Changsha 410073, China
Kalman filter to track the GNSS signals and determine the navigation solution simultaneously [7-9]. The spatial correlation between visible satellites and the positioning solution is exploited; consequently, the VDFLL performs better than conventional scalar loops, especially in environments with high dynamics and low signal power. However, the tracking sensitivity is still limited by the individual discriminators. In contrast to conventional two-step position, the direct position estimation (DPE) without discriminators was proposed in [10] and the Cramér-Rao bound was analyzed [11]. Recently, the implementing loop called maximum likelihood vector tracking loop (MLVTL) was introduced in [12]. The experiments in the indoor environment demonstrate that the benefits of the MLVTL are more pronounced than those of the VDFLL [13]. Similar to the DPE and the MLVTL, the joint acquisition combining outputs of the individual satellite correlators is used to improve the acquisition sensitivity in [14]. Both the simulation and outdoor experiments demonstrate the benefit of the joint acquisition [15,16]. However, the detection performance is not evaluated detailedly, and the collective detection degrades greatly with some blocked satellites. There are

\section{楚 Springer}

(C) 2014 Tong et al: licensee Springer. This is an Open Access article distributed under the terms of the Creative Commons

Attribution License (http://creativecommons.org/licenses/by/2.0), which permits unrestricted use, distribution, and reproduction in any medium, provided the original work is properly credited. 
some other joint acquisition algorithms, which focus on speeding up the correlator computation by combining the satellite replicas $[17,18]$. As a result, the addition of the different code replicas affects the acquisition sensitivity.

In this paper, we introduce an approach to enhance the joint acquisition for detecting the weak GNSS signals. The enhanced algorithm is characterized by the simplified architecture and the satellite selection strategy. The simplified architecture adopts the approximate calculation of the detector, which is derived in the principle of the generalized likelihood ratio test (GLRT). In the stage of performance evaluation of the simplified architecture, we derive the closed-form expressions of the probabilities of the false alarm and detection. Moreover, a satellite selection strategy is proposed to deal with the case that some satellites are blocked.

The rest of this paper is organized as follows: Firstly, we introduce the linear model for the multi-satellite signals. Secondly, the GLRT for the joint acquisition is derived and the GLRT-based architecture is presented. Thirdly, we detail the enhanced joint acquisition and the satellite selection strategy. Then, the Monte Carlo simulations are performed to validate the theoretical analysis. Finally, we conclude the paper.

\section{Multi-satellite signal model}

The GNSS signal acquisition and tracking process is a two-dimensional (code and carrier wave) signal match process in the conventional GPS receiver [1]. The acquisition detects a satellite signal and gives the initial estimate of the synchronization parameters; then, the tracking process refines the synchronization parameters. In the view of statistic signal processing, the acquisition can be modeled as the problem of detecting the deterministic signals with unknown parameters. In particular, the acquisition problem for the multi-satellite signal is simplified as

$\left\{\begin{array}{l}\mathcal{H}_{0}: x[n]=w[n] \\ \mathcal{H}_{1}: x[n]=\sum_{m=0}^{M-1} A_{m} \mathrm{~g}_{m}\left(n, \tau_{m}, f_{\mathrm{d}_{m}}\right) \cos \left(2 \pi\left(f_{\mathrm{IF}}+f_{\mathrm{d}_{m}}\right) n T_{\mathrm{s}}+\phi_{m}\right)+w[n]\end{array}\right.$

where $x[n]$ is the received digital signal, and $f_{\mathrm{IF}}$ is the intermediate frequency. $w[n]$ is the zero-mean white Gaussian noise. The sampled signal is indexed by $n$. The subscript $m$ is used to number the satellite. $T_{\mathrm{s}}$ is the sample interval. $M$ is the total number of available satellites. The pseudorandom code of the $m$ th satellite is $g_{m}(\cdot)$. The unknown parameters are listed as follows:

- $A_{m}$ : the data modulation amplitude of the $m$ th satellite signal

- $\phi_{m}$ : the carrier phase of the $m$ th satellite

- $\sigma^{2}$ : the variance of the noise $w[n]$
- $\tau_{m}$ : the signal delay of the $m$ th satellite

- $f_{\mathrm{d}_{m}}$ : the Doppler frequency of the $m$ th satellite

Hereafter, the synchronization parameters are $\tau_{m}$ and $f_{\mathrm{d}_{m}}$. In the open environment, the line-of-sight satellites are available. The total number $M$ can be determined by the assisted information such as navigation data and the rough receiver position. Some satellites are blocked and become unavailable due to local conditions (trees, buildings) in the urban environment, while line-of-sight is maintained to other satellites. These blocked satellites can be removed using the satellite selection strategy, which will be presented later. The bit transition is not considered in the signal block. Although the navigation data transition could degrade the detection performance, a number of techniques have been studied to overcome this effect [19]. Besides, the modern GNSS signals with data and pilot channels also reduce the effect of the bit transition.

\section{Derivation of the joint acquisition in principle of GLRT}

Firstly, we derive the GLRT-based detector assuming that the synchronization parameters are known in this section. Secondly, we review the asymptotic maximum likelihood estimator (MLE) of the synchronization parameters based on the position searching. Finally, the implementing architecture of the joint acquisition is detailed.

\section{Known synchronization parameters}

It is assumed that Doppler frequency and delay have been estimated; then, the unknown parameter set is $\left\{\mathbf{A}, \boldsymbol{\phi}, \sigma^{2}\right\}$. Similar to $[20,21]$, the signal $x[n]$ can be transformed to be linear in $A_{m}$ and $\phi_{m}$ by letting

$$
\left\{\begin{array}{l}
\alpha_{m}=A_{m} \cos \phi_{m} \\
\beta_{m}=A_{m} \sin \phi_{m}
\end{array}\right.
$$

Then, the linear model is given by

$$
\mathbf{x}=\mathbf{H} \boldsymbol{\theta}+\mathbf{w}
$$

where

$$
\begin{aligned}
\mathbf{x}_{N \times 1} & =[x[0], x[1], \ldots, x[N-1]]^{T} \\
\boldsymbol{\theta}_{2 M \times 1} & =\left[\alpha_{0}, \beta_{0}, \ldots, \alpha_{M-1}, \beta_{M-1}\right]^{T} \\
\mathbf{w}_{N \times 1} & =[w[0], w[1], \ldots, w[N-1]]^{T} \\
\mathbf{H}_{n, i} & =\left\{\begin{array}{l}
g_{m}\left(n, \tau_{m}, f_{\mathrm{d}_{m}}\right) \cos \left(2 \pi\left(f_{\mathrm{IF}}+f_{\mathrm{d}_{m}}\right) n T_{\mathrm{s}}\right), i=2 m \\
g_{m}\left(n, \tau_{m}, f_{\mathrm{d}_{m}}\right) \sin \left(2 \pi\left(f_{\mathrm{IF}}+f_{\mathrm{d}_{m}}\right) n T_{\mathrm{s}}\right), i=2 m+1
\end{array}\right.
\end{aligned}
$$

with $n=0,1, \ldots, N-1$ and $m=0,1, \ldots, M-1$.

The hypothesis testing model in (1) is equivalent to the classical linear model

$$
\begin{aligned}
& \mathcal{H}_{0}: \boldsymbol{\theta}=\mathbf{0} \\
& \mathcal{H}_{1}: \boldsymbol{\theta} \neq \mathbf{0}
\end{aligned}
$$


with unknown $\sigma^{2}$. According to Theorem 9.1 of [20], the GLRT for the model in (4) decides $\mathcal{H}_{1}$ if

$$
T(\mathbf{x})=\frac{N-2 M}{2 M} \cdot \frac{\hat{\boldsymbol{\theta}}_{1}^{T} \mathbf{H}^{T} \mathbf{H} \hat{\boldsymbol{\theta}}_{1}}{\mathbf{x}^{T}\left(\mathbf{I}-\mathbf{H}\left(\mathbf{H}^{T} \mathbf{H}\right)^{-1} \mathbf{H}^{T}\right) \mathbf{x}}>\gamma
$$

where $\hat{\boldsymbol{\theta}}_{1}=\left(\mathbf{H}^{T} \mathbf{H}\right)^{-1} \mathbf{H}^{T} \mathbf{x}$ is the unrestricted MLE of $\boldsymbol{\theta}$ under $\mathcal{H}_{1}$ and $\gamma$ is the detection threshold. The probability distribution function (PDF) of the statistic $T(\mathbf{x})$ is [20]

$$
T(\mathbf{x}) \sim \begin{cases}F_{2 M, N-2 M} & \text { under } \mathcal{H}_{0} \\ F_{2 M, N-2 M}^{\prime}(\lambda) & \text { under } \mathcal{H}_{1}\end{cases}
$$

where $F_{2 M, N-2 M}$ is an $F$ distribution with $2 M$ and $N-2 M$ degrees of freedom. The $F_{2 M, N-2 M}^{\prime}$ is a noncentral $F$ distribution with $2 M$ and $N-2 M$ degrees of freedom and noncentrality parameter $\lambda$.

The statistical distributions given in (6) converge to $\chi^{2}$ distributions as $N \rightarrow \infty$ [20]. For the multi-satellite signal model (1), the number $N$ is larger than 2,000 when the GPS C/A codes are used and the IF digital signal persists for $1 \mathrm{~ms}$. In this case, we have the asymptotic distributions of the modified statistic $T^{\prime}(\mathbf{x})$ satisfying

$$
T^{\prime}(\mathbf{x})=2 M \cdot T(\mathbf{x}) \sim \begin{cases}\chi_{2 M}^{2} & \text { under } \mathcal{H}_{0} \\ \chi_{2 M}^{\prime 2}(\lambda) & \text { under } \mathcal{H}_{1}\end{cases}
$$

where $\chi_{2 M}^{2}$ denotes a chi-squared distribution with $2 M$ degrees of freedom and $\chi_{2 M}^{\prime 2}(\lambda)$ denotes a noncentral chisquared distribution with $2 M$ degrees of freedom and noncentrality parameter $\lambda$. Herein, $\lambda$ is the same as that shown in (6) and can be calculated by

$$
\lambda=\frac{\boldsymbol{\theta}_{1}^{T} \mathbf{H}^{T} \mathbf{H} \boldsymbol{\theta}_{1}}{\sigma^{2}}
$$

where $\boldsymbol{\theta}_{1}$ is true value of $\boldsymbol{\theta}$ under $\mathcal{H}_{1}$. The unbiased estimator of the $\sigma^{2}$ is given by [20]

$$
\hat{\sigma}^{2}=\frac{\mathbf{x}^{T} \mathbf{x}-\mathbf{x}^{T} \mathbf{H}\left(\mathbf{H}^{T} \mathbf{H}\right)^{-1} \mathbf{H}^{T} \mathbf{x}}{N-2 M}
$$

With the substitution of (5) in (7), the equivalent GLRT decides $\mathcal{H}_{1}$ if

$$
T^{\prime}(\mathbf{x})=(N-2 M) \cdot \frac{\mathbf{x}^{T} \mathbf{H}\left(\mathbf{H}^{T} \mathbf{H}\right)^{-1} \mathbf{H}^{T} \mathbf{x}}{\mathbf{x}^{T} \mathbf{x}-\mathbf{x}^{T} \mathbf{H}\left(\mathbf{H}^{T} \mathbf{H}\right)^{-1} \mathbf{H}^{T} \mathbf{x}}>\gamma^{\prime}
$$

where the detection threshold $\gamma^{\prime}=2 M \gamma$ and satisfies

$$
P_{\mathrm{fa}}=\int_{\gamma^{\prime}}^{\infty} \frac{1}{(M-1) ! 2^{M}} t^{M-1} \exp \left(-\frac{1}{2} t\right) d t
$$

The detection probability is

$P_{\mathrm{d}}=\int_{\gamma^{\prime}}^{\infty} \frac{1}{2}\left(\frac{t}{\lambda}\right)^{\frac{M-1}{2}} \exp \left(-\frac{1}{2}(t+\lambda)\right) I_{M-1}(\sqrt{\lambda t}) d t$

where $I_{M-1}(\cdot)$ is the modified Bessel function of the first kind.

The detector in (10) is also termed as the constant false alarm rate (CFAR) detector. If $M=1$, it is easily obtained that the modified statistic test (10) is equivalent to the coherent detector in conventional acquisition [21]. The conventional scalar acquisition searches the visible satellites separately, while the joint acquisition tries to combine the coherent integration results of different satellites noncoherently.

\section{Unknown synchronization parameters}

The Doppler frequency and delay are always unknown in the GNSS receiver before successful acquisition. Recall that the matrix $\mathbf{H}$ is a function of $\boldsymbol{\tau}$ and $\mathbf{f}_{d}$ in (3). As derived in [10], the MLE of synchronization parameters can be obtained by maximizing the likelihood function, which is equivalent to minimizing the nonlinear cost function

$$
\begin{aligned}
\hat{\tau}, \hat{f_{d}} & =\arg \min _{\tau, f_{d}} \frac{1}{N}\|\mathbf{x}-\mathbf{H} \boldsymbol{\theta}\|^{2} \\
& =\arg \min _{\tau, f_{d}}\left\{\frac{\mathbf{x}^{T} \mathbf{x}-\mathbf{x}^{T} \mathbf{H}\left(\mathbf{H}^{T} \mathbf{H}\right)^{-1} \mathbf{H}^{T} \mathbf{x}}{N}\right\} \\
& =\arg \max _{\tau, f_{d}}\left\{T^{\prime}\left(\mathbf{x}, \tau, f_{d}\right)\right\}
\end{aligned}
$$

The problem of interest is to make full use of the constrained relation of different synchronization parameters to obtain more processing gain. Notice that the projection from user motion parameters to the synchronization parameters is injective when the total number of the available satellites is no less than 4. Hence, combining the residual satellites will provide more processing gain. As presented in [10], the MLE of the synchronization parameters is equivalent to the MLE of the user motion parameters

$$
\begin{aligned}
\hat{\Upsilon} & =\arg \min _{\Upsilon}\{J(\Upsilon)\} \\
& =\arg \max _{\Upsilon}\left\{T^{\prime}(\mathbf{x}, \Upsilon)\right\}
\end{aligned}
$$

where $\Upsilon=\left[\mathbf{p}^{\mathbf{T}}, \mathbf{v}^{\mathbf{T}}, \delta \mathbf{t}^{\mathbf{T}}\right]$ is the extended user motion parameters. $\mathbf{p}=[x, y, z]^{T}$ is the position of the user in the Earth-centered Earth-fixed (ECEF) coordinate system. Similarly, $\mathbf{v}=\left[v_{x}, v_{y}, v_{z}\right]^{T}$ is the velocity of the user in the ECEF coordinate system. $\delta \mathbf{t}=[\delta t, \dot{\delta} t]$ is the receiver clock bias and clock drift, respectively. As described in $[1,10]$, the projections from the extended user motion 
parameters $\Upsilon$ to the synchronization parameters are given by

$$
\begin{gathered}
\tau_{m}=\frac{\left\|\mathbf{p}^{(m)}-\mathbf{p}\right\|}{c}+\delta t-\delta t^{(m)}+\tau_{I}^{(m)}+\tau_{T}^{(m)} \\
f_{\mathrm{d}_{m}}=\frac{f_{\mathrm{c}}}{c} \frac{\mathbf{p}^{(m)}-\mathbf{p}}{\left\|\mathbf{p}^{(m)}-\mathbf{p}\right\|}\left(\mathbf{v}^{(m)}-\mathbf{v}\right)+\delta \dot{t}-\dot{\delta} t^{(m)}
\end{gathered}
$$

where $c$ is the speed of light, $\mathbf{p}^{(m)}$ is the Sagnac-corrected position of the $m$ th satellite in ECEF coordinate system, $\delta t^{(m)}$ is the clock bias in seconds for the $m$ th satellite, and $\tau_{I}^{(m)}$ and $\tau_{T}^{(m)}$ are the tropospheric delay and ionospheric delay in seconds, respectively. In (16), $f_{\mathrm{c}}$ is the carrier frequency, $\mathbf{v}^{(m)}$ is the velocity of the $m$ th satellite, and $\dot{\delta} t^{(m)}$ is the clock drift in units of hertz for the $m$ th satellite. As the annotation in (1), $\tau_{m}$ and $f_{\mathrm{d}_{m}}$ refer to the code delay and carrier Doppler frequency, respectively.

With substitution of (13) and (14) in (10), the final GLRT of the model in (3) with unknown delay and Doppler frequency decides $\mathcal{H}_{1}$ if

$$
\begin{aligned}
T^{\prime}\left(\mathbf{x} ; \hat{\boldsymbol{\tau}}, \hat{\mathbf{f}}_{\mathrm{d}}\right) & =\max _{\boldsymbol{\tau}, \mathbf{f}_{\mathrm{d}}} T^{\prime}\left(\mathbf{x} ; \boldsymbol{\tau}, \mathbf{f}_{\mathrm{d}}\right) \\
& =\max _{\Upsilon} T^{\prime}(\mathbf{x} ; \Upsilon)>\gamma^{\prime}
\end{aligned}
$$

for all possible $\Upsilon$. The threshold $\gamma^{\prime}$ is determined by (11). The number of the search bins depends on the quality of the initial user position provided by the assisted information. The resolutions of the position and velocity for acquisition can be lower than those for tracking. However, the computation load cannot be neglected. The $2 M \times 2 M$ dimensional inverse matrix is calculated in the individual statistic shown in (10), the computation load increases significantly if more satellites are available.

\section{The implementing architecture}

An implementing architecture of the joint acquisition is shown in Figure 1. Whereas the traditional acquisition performs two-dimensional search over the delay and Doppler frequency for each satellite, the joint acquisition conducts multi-dimensional search over the receiver motion parameters and the clock errors. The search space center for the joint acquisition consists of the rough receiver position, velocity, and time (PVT), which are provided by the assisted information. The resolution of search bins is related to the quality of the search space center. Another way to determine the solution is dividing the search space into some subspaces with different solutions [12].

As shown in Figure 1, all the local signals are generated individually by the synchronization parameters $\boldsymbol{\tau}$ and $\mathbf{f}_{\mathrm{d}}$. As described in (15) and (16), these synchronization parameters are determined by the searching bin. The replica local signals appear in complex form in Figure 1. The real parts of the complex signals correspond to the even columns of the matrix $\mathbf{H}$ in (3), while the imaginary parts correspond to the odd columns. The coherent integration at individual channel is implemented after the carrier and code stripping process. Then, the integrated results, quadratic sum of signals, and the matrix $\mathbf{H}$ form the statistic in (10). The MLE of the $\Upsilon, \hat{\Upsilon}$ is obtained by maximizing the statistics over the search space. The joint acquisition is successful if the maximum statistic

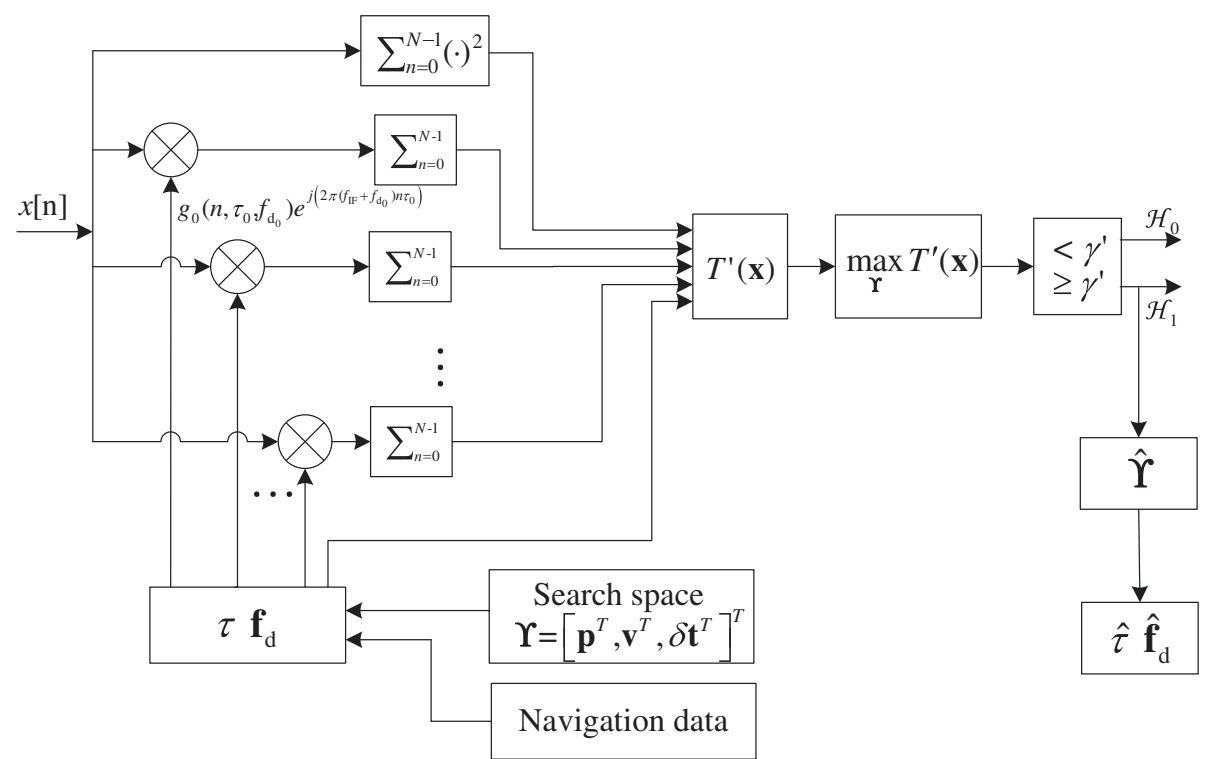

Figure 1 The implementing architecture for the joint acquisition. 
exceeds the threshold $\gamma^{\prime}$ in (17); then, the estimated synchronization parameters of all the available satellites are obtained, respectively. Some implementation losses may exist as a result of residual errors in the replicas of the estimated synchronization parameters. Thus, the detection probability in (12) should be viewed as the upper bound.

The implementing architecture depicted in Figure 1 is similar to the MLE-based tracking architecture named MLVTL in [12]. There are, however, two differences. One is that the resolution of the search bins in Figure 1 is lower than that in the MLVTL. Besides, the scope of the search space for the joint acquisition is wider than that for the MLVTL. As a result, the joint acquisition produces the approximate MLE of the receiver motion parameters and clock errors while the MLVTL generates the refined estimates. The other one is that the joint acquisition decides whether the signals are strong enough to detect. If all the signals are degraded seriously or even blocked, the MLE of the receiver motion parameters still can be obtained by the joint acquisition and the MLVTL. The joint acquisition declares that the estimated values are noneffective by checking the statistic while the MLVTL generates the distorted results. It is suggested that the statistic $T^{\prime}(\mathbf{x})$ be used as an indicator for the lock state of the MLE-based tracking loop.

\section{The enhanced architecture for joint acquisition}

Acquiring the GNSS signals together, rather than individually, improves the acquisition sensitivity. However, the drawbacks of the joint acquisition are obvious. As discussed above, the calculation of the matrix inverse is extensive and the computation load is heavy. When some satellites are blocked, the final statistic reduces dramatically while the threshold is invariable. Thus, the performance of the joint acquisition is degraded. To overcome these drawbacks, a simplified architecture of the joint acquisition with the satellite selection strategy is proposed.

\section{A simplified implementing architecture}

As discussed in the previous section, the computation load of the joint acquisition increases dramatically with the satellite number due to the $2 M \times 2 M$ dimensional matrix inverse. Comparing (9) in [10] with (13) in this paper, we find that $R_{d d}$ in [10] corresponds to the $\mathbf{H}^{T} \mathbf{H}$ in (13). The term $R_{d d}$ denotes the correlation of the spreading codes, including the autocorrelations and cross-correlations. In GPS signals, the cross-correlation level of the $\mathrm{P}(\mathrm{Y})$ codes with any other $\mathrm{P}(\mathrm{Y})$ code approaches $-127 \mathrm{~dB}$ with respect to maximum autocorrelation. Therefore, the cross-correlation of the $\mathrm{P}(\mathrm{Y})$ codes can be neglected. The cross-correlation level of the GPS
$\mathrm{C} / \mathrm{A}$ codes can be as poor as $-21.1 \mathrm{~dB}$ in the case of high Doppler difference [1]. The empirical cross-correlation mean value of Galileo E1 codes is about $-24.9 \mathrm{~dB}$ [18]. However, the GNSS signals are below the noise floor and the available satellite number is finite. The effect of the cross-correlations is not too severe. The quantified power loss caused by the cross-correlations can be determined by $C_{m} / N_{0}$ and $\left(C_{m} / N_{0}\right)_{\text {eff }}[1]$

$$
\begin{aligned}
L_{\mathrm{eff}, \mathrm{dB}} & =C_{m} / N_{0}-\left(C_{m} / N_{0}\right)_{\mathrm{eff}} \\
& =10 \log _{10}\left(1+\frac{1}{Q R_{c}} \sum_{k=0, k \neq m}^{M-1} 10^{\frac{C_{k} / N_{0}}{10}}\right)
\end{aligned}
$$

where $L_{\mathrm{eff}, \mathrm{dB}}$ is the effective signal power loss in decibels, $Q=1.5$ and $R_{c}=1.023 \mathrm{MHz}$ with the $\mathrm{C} / \mathrm{A}$ code, and $\left(C_{m} / N_{0}\right)_{\text {eff }}$ is the effective signal power to noise density of the $m$ th satellite. Both $C_{m} / N_{0}$ and $\left(C_{m} / N_{0}\right)_{\text {eff }}$ are in decibel hertz.

To evaluate the effect of multiple-access interference, it is assumed that all the signals from different satellites have the same power. The effective signal power losses changing with $C / N_{0}$ under different numbers of available satellites are shown in Figure 2. It is clear to see that the effective signal losses caused by the cross-correlations are less than $0.5 \mathrm{~dB}$ even with 16 available satellites when $C / N_{0}$ is less than $42 \mathrm{~dB}-\mathrm{Hz}$. $\left(C / N_{0}\right)_{\text {eff }}$ reduces slightly with $M=4$. Even in the worst case, the degraded level is less than $1.5 \mathrm{~dB}$ with $M=16$ and $C / N_{0}=46 \mathrm{~dB}$ $\mathrm{Hz}$. Typically, the nominal signal power of GPS C/A code is $-158.5 \mathrm{dBW}$ [1], which is equivalent to $45 \mathrm{~dB}-\mathrm{Hz}$ for most receivers. Therefore, it is reasonable to ignore the effect of the cross-correlations in the joint acquisition for the weak signals.

Neglecting the cross-correlation between the spreading codes, we calculate the matrix inverse in (13) approximately

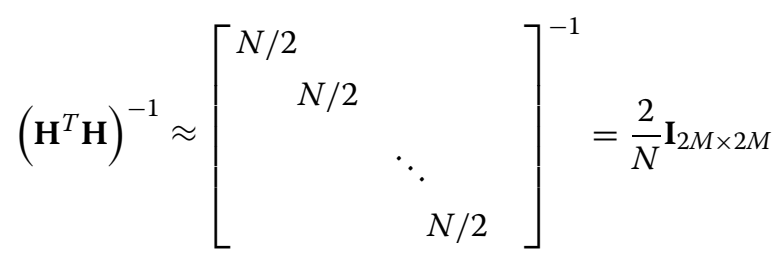

where $\mathbf{I}_{2 M \times 2 M}$ denotes the $2 M \times 2 M$ identity matrix.

The detector of the joint acquisition is simplified further by substituting (19) into (10)

$$
\begin{aligned}
T^{\prime}\left(\mathbf{x} ; \boldsymbol{\tau}, \mathbf{f}_{\mathrm{d}}\right) & \approx(N-2 M) \cdot \frac{\frac{2}{N} \mathbf{x}^{T} \mathbf{H} \mathbf{H}^{T} \mathbf{x}}{\mathbf{x}^{T} \mathbf{x}-\frac{2}{N} \mathbf{x}^{T} \mathbf{H H}^{T} \mathbf{x}} \\
& =(N-2 M) \cdot \frac{I_{M}\left(\boldsymbol{\tau}, \mathbf{f}_{\mathrm{d}}\right)}{\mathbf{x}^{T} \mathbf{x}-I_{M}\left(\boldsymbol{\tau}, \mathbf{f}_{\mathrm{d}}\right)}
\end{aligned}
$$




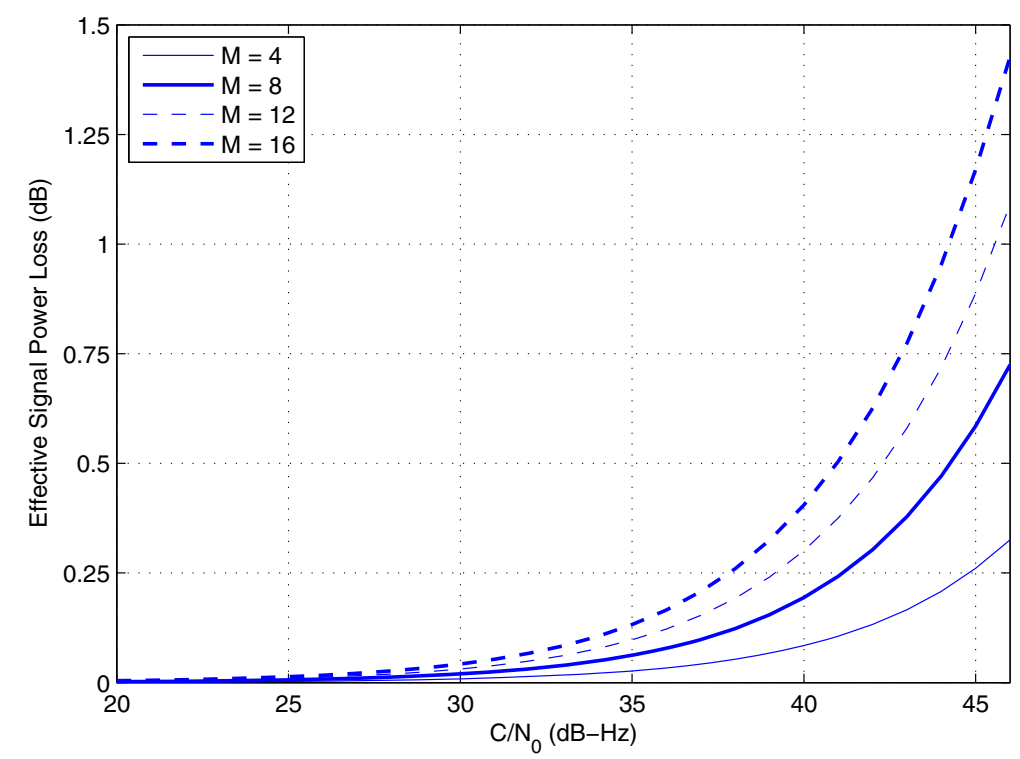

Figure 2 The effective signal power loss caused by the cross-correlation. The dashed line denotes the results with $M=12$. The dashed bold line denotes the results with $M=16$.

where $I_{M}\left(\boldsymbol{\tau}, \mathbf{f}_{\mathrm{d}}\right)$ is defined by

$$
\begin{aligned}
I_{M}\left(\boldsymbol{\tau}, \mathbf{f}_{\mathrm{d}}\right) & =\frac{2}{N} \mathbf{x}^{T} \mathbf{H} \mathbf{H}^{T} \mathbf{x} \\
& =\frac{2}{N} \sum_{m=0}^{M-1}\left|\sum_{n=0}^{N-1} x[n] \mathrm{g}_{m}\left(n, \tau_{m}, f_{\mathrm{d}_{m}}\right) \mathrm{e}^{\mathrm{j}\left(2 \pi\left(f_{\mathrm{IF}}+f_{\mathrm{d}_{m}}\right) n T_{\mathrm{s}}\right)}\right|^{2} \\
& =\sum_{m=0}^{M-1} I_{m}\left(\tau_{m}, f_{d_{m}}\right)
\end{aligned}
$$

It can be concluded from (21) that the $I_{m}\left(\tau_{m}, f_{d_{m}}\right)$ is the coherent integration results of the $m$ th satellite signals, while $I_{M}\left(\boldsymbol{\tau}, \mathbf{f}_{\mathrm{d}}\right)$ is the noncoherent sum of $I_{m}\left(\tau_{m}, f_{d_{m}}\right)$.

Similarly, the noncentral parameter $\lambda$ is approximated by

$$
\begin{aligned}
\lambda & \approx \frac{N \boldsymbol{\theta}_{1}^{T} \boldsymbol{\theta}_{1}}{2 \sigma^{2}} \\
& =N \sum_{m=0}^{M-1} \frac{A_{m}^{2}}{2 \sigma^{2}} \\
& =2 T_{\operatorname{coh}} \sum_{m=0}^{M-1} 10^{\frac{C_{m} / N_{0}}{10}}
\end{aligned}
$$

where $T_{\text {coh }}$ is the coherent integration time.

The implementing architecture of the simplified joint acquisition is shown in Figure 3. One notable difference between the GLRT-based architecture in Figure 1 and the simplified architecture in Figure 3 is the calculation of $T^{\prime}(\mathbf{x})$. As shown in Figure 3, the coherent integration results of the individual satellites are summed noncoherently to form $I_{M}\left(\boldsymbol{\tau}, \mathbf{f}_{\mathrm{d}}\right)$. The inverse computation of the $2 M \times 2 M$ dimensional matrix is avoided. Furthermore, it is convenient to switch back and forth between the simplified joint acquisition and the conventional acquisition with the individual integration results $I_{m}\left(\tau_{m}, f_{d_{m}}\right)$. The other difference between the two implementing architectures is whether the blocked satellites are identified and removed. The satellite selection strategy to enhance the simplified joint acquisition will be presented in the next section.

\section{Satellite selection strategy}

In the conventional acquisition, there is a binary hypothesis test per satellite signal with associated probabilities of false alarm and detection. The two-dimensional search over the Doppler and delay is performed to form the detector individually. The conventional acquisition is still effective with and without $a$ priori information, such as ephemeris, initial receiver position, and so on. When these information are available in the reacquisition or the assistant GNSS, the limited process gain can be obtained by reducing the search scope and fining the searching solution in the conventional acquisition. Furthermore, the joint acquisition combines all these binary hypothesis tests into a single test to obtain more process gain. In the open ground, the line-of-sight satellites to be acquired are easily determined by the ephemeris and initial position. However, the set of available satellites cannot be directly determined by a priori information in the cases where some satellite signals are blocked by the buildings, 


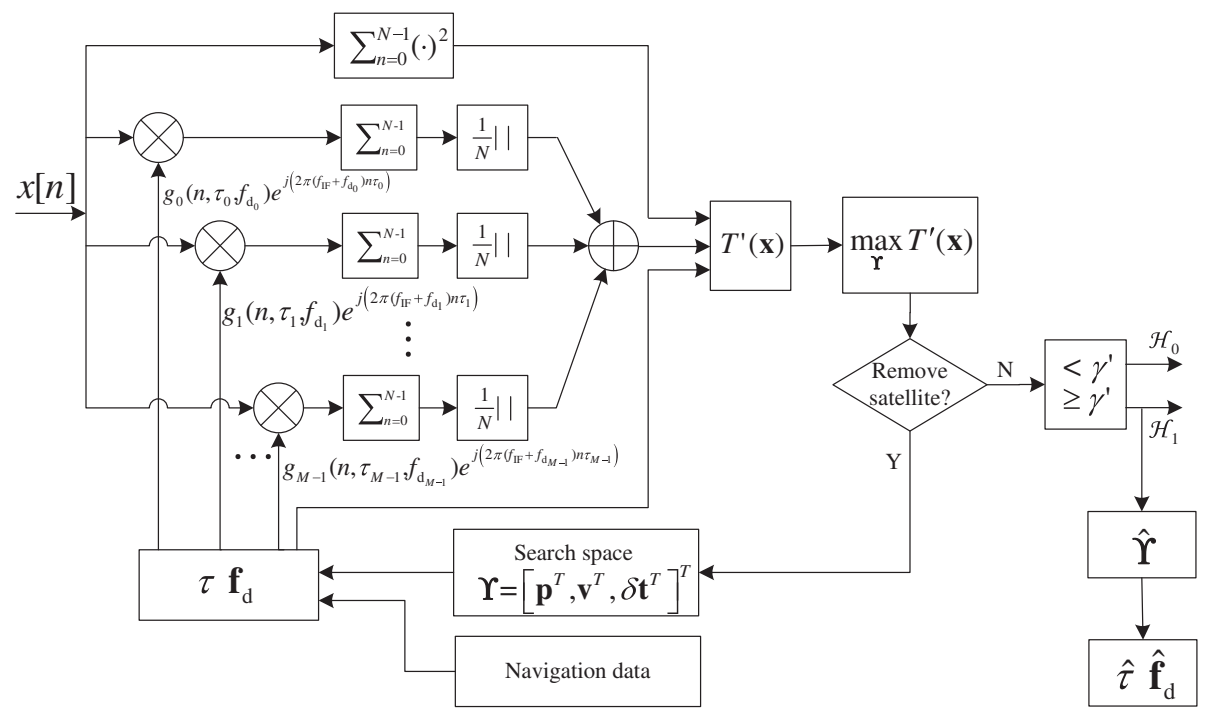

Figure 3 The enhanced architecture for the joint acquisition.

mountains, or trees. Under this condition, the blocked satellites are still regarded as available in the joint acquisition. It is concluded from (22) that the probability of detection decreases considerably with the blocked satellites. To deal with the degraded or blocked satellites, the satellite selection is proposed in this section.

As shown in Figure 3, the satellite selection strategy is performed after the maximum $T^{\prime}(\mathbf{x})$. The satellites which are regarded as unavailable will be removed from the set of light-of-sight satellites. Then, a research process is implemented to find the maximum $T^{\prime}(\mathbf{x})$ with the updated set of light-of-sight satellites. The key point of the selection strategy is how to judge the satellite is blocked or unavailable. Recall the statistic in (20) and (21); it can be found that the statistic of the joint acquisition is the sum of the individual statistic per satellite. The statistic of the $m$ th satellite signal is

$$
T^{\prime(m)}(\mathbf{x})=\frac{I_{m}\left(\tilde{\tau}_{m}, \tilde{f}_{d_{m}}\right)}{\hat{\sigma}^{2}}
$$

where $\tilde{\tau}_{m}$ and $\tilde{f}_{d_{m}}$ correspond to the motion parameter $\tilde{\Upsilon}$, which is obtained by maximizing $T^{\prime}(\mathbf{x})$ as shown in Figure 3. Another research process will be performed if some satellites are removed, so $\tilde{\tau}_{m}$ and $\tilde{f}_{d_{m}}$ may be different from the final estimates $\left(\hat{\tau}_{m}\right.$ and $\left.\hat{f}_{d_{m}}\right)$. Considering independence of the detector per satellite signal, the distribution of the individual statistic is

$$
T^{\prime(m)}(\mathbf{x}) \sim \begin{cases}\chi_{2}^{2} & \text { under } \mathcal{H}_{0} \\ \chi_{2}^{\prime 2}\left(\lambda^{(m)}\right) & \text { under } \mathcal{H}_{1}\end{cases}
$$

where $\lambda^{(m)}$ is defined in (22). If the signal from the $m$ th satellite is blocked, the signal power becomes attenuated and the parameter $\lambda^{(m)}$ approximates to zero. Otherwise, $\lambda^{(m)}$ is larger than zero and increases with the signal power. The estimates of $\lambda^{(m)}$ and $C / N_{0}$ for the available satellite are rough during the acquisition process, whereas the estimates for the seriously blocked satellite are almost the same in acquisition and tracking process. The refined estimate of $\lambda^{(m)}$ is calculated as follows:

$$
\begin{aligned}
\hat{\lambda}^{(m)} & =E\left[T^{\prime(m)}(\mathbf{x})\right]-2 \\
& \approx \frac{1}{K} \sum_{k=1}^{K} T_{k}^{\prime(m)}(\mathbf{x})-2
\end{aligned}
$$

where $K$ should be large enough, herein $K=1,000$. The expectation and variance of $\hat{\lambda}^{(m)}$ are

$$
\begin{aligned}
E\left[\hat{\lambda}^{(m)}\right] & =\lambda^{(m)} \\
\sigma^{2}\left(\hat{\lambda}^{(m)}\right) & =\frac{4\left(1+\lambda^{(m)}\right)}{K}
\end{aligned}
$$

If the estimated $\hat{\lambda}^{(m)}$ is less than the threshold $\lambda_{\text {th }}$, the $m$ th satellite is regarded as blocked. Otherwise, the $m$ th satellite is available. The threshold $\lambda_{\text {th }}$ can be determined by the rule of thumb. Another conservative way to determine the threshold is assuming that all the signals have the same power. The minimum $\lambda^{(m)}$ for a given $P_{\mathrm{d}}$ is regarded as the threshold $\lambda_{\text {th }}$, which satisfies

$$
\begin{aligned}
P_{\mathrm{d}}= & \int_{\gamma^{\prime}}^{\infty} \frac{1}{2}\left(\frac{t}{M \lambda_{\mathrm{th}}}\right)^{\frac{M-1}{2}} \exp \left(-\frac{1}{2}\left(t+M \lambda_{\text {th }}\right)\right) \\
& \times I_{M-1}\left(\sqrt{M \lambda_{\text {th }} t}\right) d t
\end{aligned}
$$

where $M$ is the total satellite number used in the search process and $\gamma^{\prime}$ is determined by (11). 
All the maximum values of the individual statistics are consistent with the true PVT in the normal circumstance without interference signals. However, the multipath interference may exist in the blocked environments. The multipath will distort the consistence between the individual statistics and the true PVT, which can result in the outliers of the estimated Doppler and delay. How to detect and remove the multiple outliers may refer to [22]. We only focus on the direct-path signals in this paper.

\section{Simulation results}

\section{Validation and comparison of the detection performance}

The Monte Carlo simulations are conducted to validate the analytic results. Through all simulations, the intermediate frequency (IF) $f_{\mathrm{IF}}$ is $4.068 \mathrm{MHz}$ and the sampling frequency $f_{\mathrm{s}}$ is $6.140625 \mathrm{MHz}$. The IF bandwidth is $f_{\mathrm{s}} / 2$. The GPS C/A codes are used as the PRN codes of satellites. The number of the available satellites in different scenarios are $1,4,8,12$, and 16 , respectively. The front-end filter has been neglected. The coherent integration time is $1 \mathrm{~ms}$. The navigation data and rough receiver position are provided as a priori information.

The statistic of the joint acquisition is the multivariate function, so it is assumed that the receiver positions only in the $X$ axis and $Y$ axis is unknown to show the three-dimensional function. The total satellite number is 12 in this scenario. Figure 4 shows the GLRTbased $T^{\prime}(\mathbf{x})$ in (10) calculated from different errors in the $X$ axis and $Y$ axis, denoted as $\Delta x$ and $\Delta y$. Similarly, the simplified statistics $T^{\prime}(\mathbf{x})$ defined by (20) are shown in Figure 5. It is clear to see that the GLRT-based statistic and the simplified statistic performs similarly with different $\Delta x$ and $\Delta y$. Both the statistics have the maximum values at the right position. The maximum amplitude of the simplified statistic decreases slightly due to the cross-correlation of the C/A codes, which means that the detection performance of the GLRTbased statistic is little better than those of the simplified statistic.

For simplicity and clarity, carrier power to noise density $C / N_{0}$ per satellite is equal to each other. The weak signals with $C / N_{0}=30 \mathrm{~dB}-\mathrm{Hz}$ are used for different acquisition methods. The estimated receiver operating characteristic (ROC) curves, as shown in Figure 6, are estimated by the conventional scalar acquisition and the joint acquisition combining 4, 8, 12, and 16 satellites, respectively. For comparison, the theoretical curves derived in previous section are also plotted in Figure 6. It is obvious to see that the numeric results are always consistent with the theoretical curves. In all considered cases, the results of the proposed algorithm perform markedly better than those of the scalar acquisition. Moreover, the performance of the joint acquisition is steadily improved as available satellites are becoming more. This is because the joint acquisition makes full use of the correlation between the multi-satellite signals.

Further simulations are implemented to demonstrate the acquisition performance. As shown in Figure 7, the false alarm probability is fixed to 0.001 . The probability of detection changes with $C / N_{0}$ ranging from 20 to

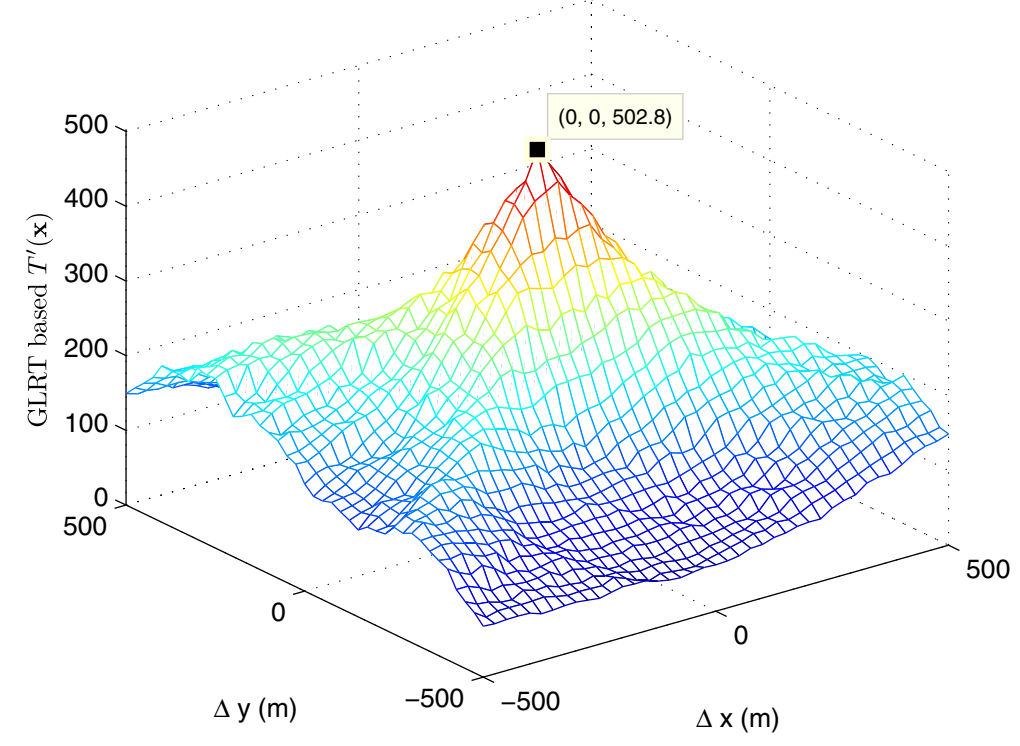

Figure 4 The GLRT-based statistic. 


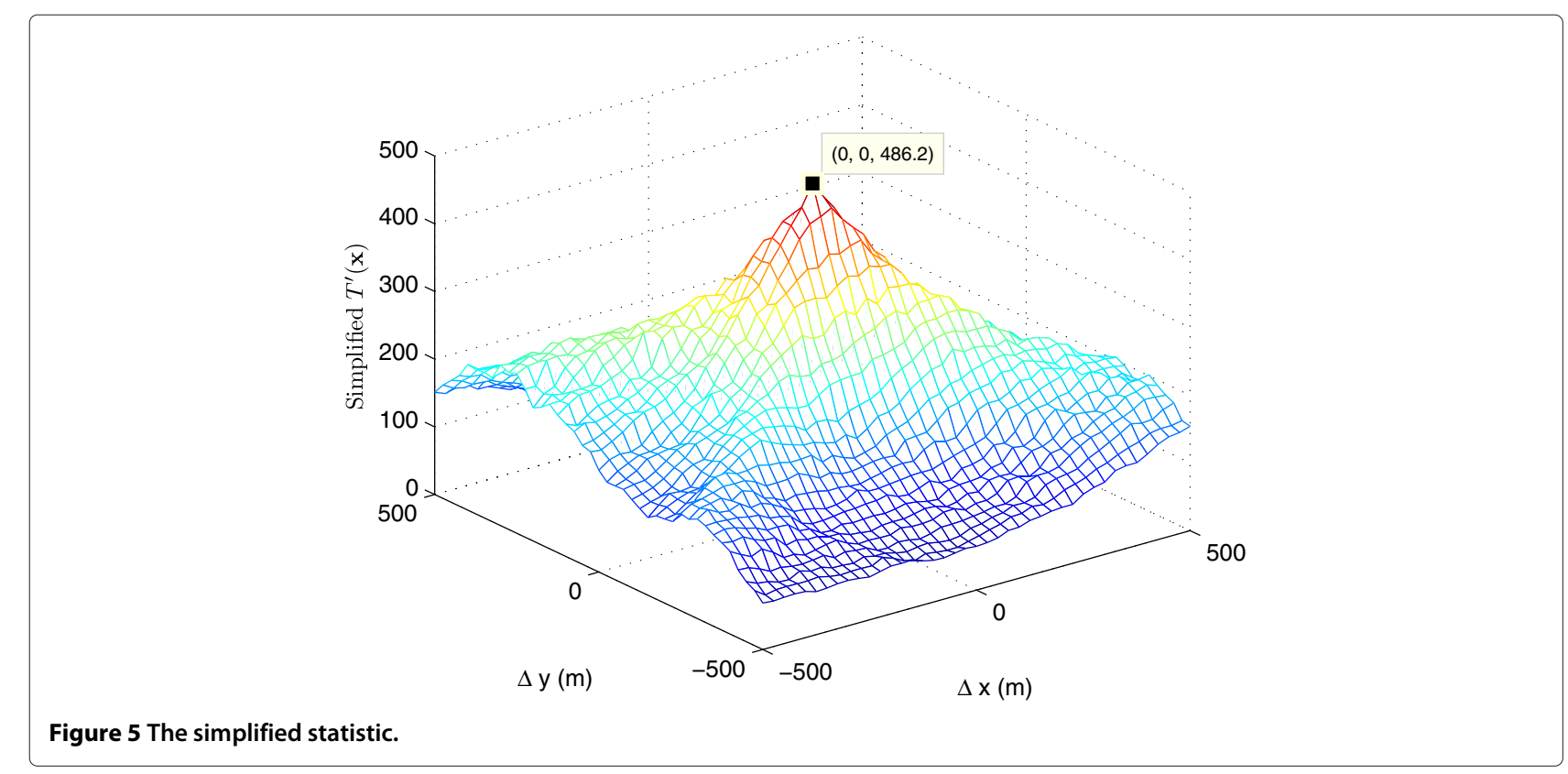

$50 \mathrm{~dB}-\mathrm{Hz}$. The analytic detection probability is also validated by numeric results. Besides, it is clear to see that the joint acquisition with the most satellites outperforms the other methods. Given the probability of detection $P_{\mathrm{d}}=90 \%$, the lowest $C / N_{0}$ is $32 \mathrm{~dB}-\mathrm{Hz}$ for the joint acquisition with 16 satellites, while the lowest $C / N_{0}$ is about $41 \mathrm{~dB}-\mathrm{Hz}$ for the conventional acquisition.

\section{Demonstration of the benefits of satellite selection}

The special scenarios are considered to demonstrate the enhancement of the satellite selection strategy. In these scenarios, 12 satellites should be available. However, only normal four satellites are considered to be always in line of sight. The normal signals satisfies $C / N_{0}=36 \mathrm{~dB}-\mathrm{Hz}$. The signals from the other eight satellites are regarded as degraded or blocked. The degraded satellites are assumed to have the same signal power. The $C / N_{0}$ of abnormal signals changes from 10 to $36 \mathrm{~dB}-\mathrm{Hz}$ in different scenarios. The probabilities of detection evaluated by the simplified joint acquisition (JA) with and without the satellites selection strategy are shown in Figure 8. The axis label $C / N_{0}$ denotes the carrier power to noise density of the abnormal signals. It is obvious that the simplified joint

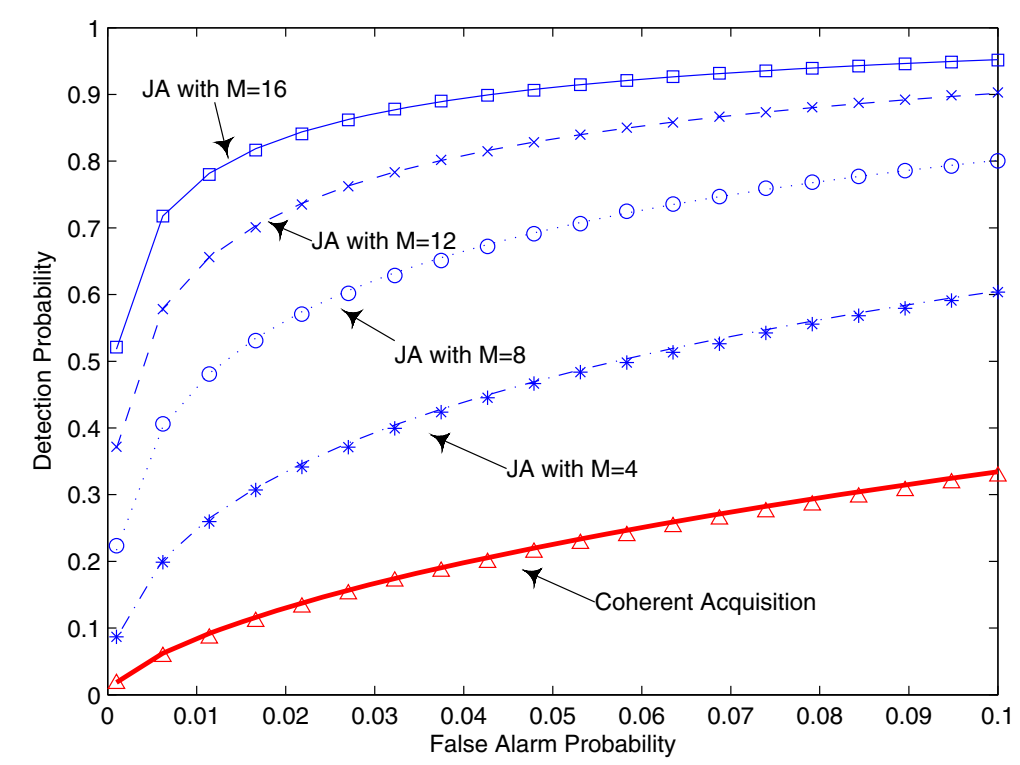

Figure 6 The ROC comparison among different acquisition methods. 


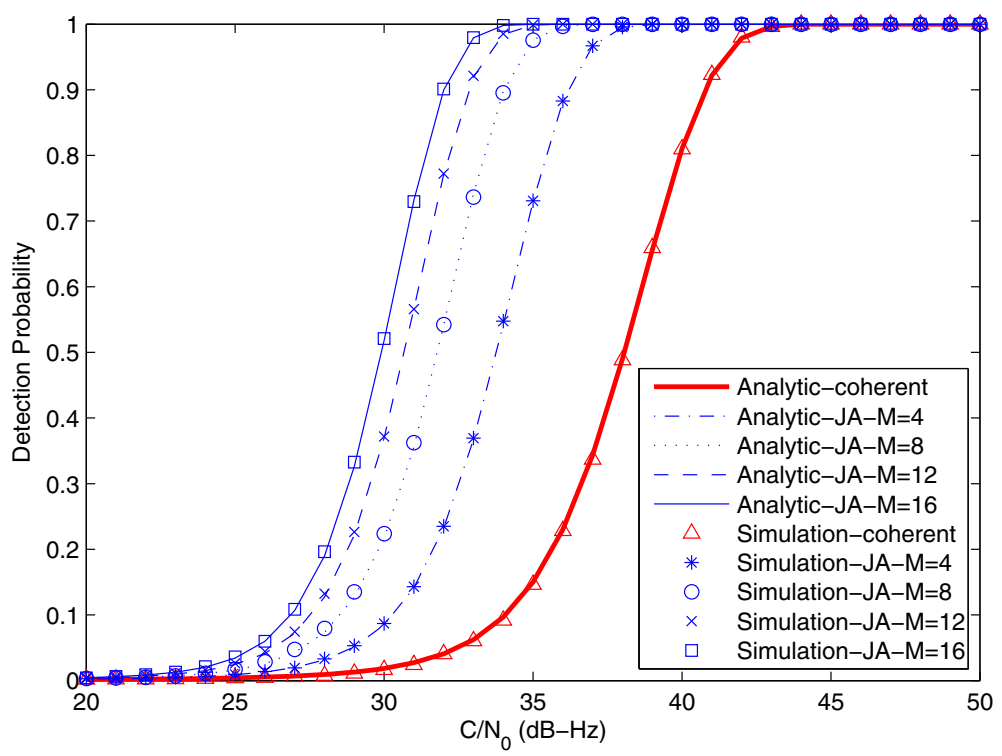

Figure 7 The detection probabilities with different $C / N_{0}$. The red bold line denotes the analytic results of the traditional acquisition. The dash-dot line denotes the analytic results of the joint acquisition with $M=4$. The dash-dot line denotes the analytic results of the joint acquisition with $M=8$. The dashed line denotes the analytic results of the joint acquisition with $M=12$. The solid line denotes the analytic results of the joint acquisition with $M=16$. The red diamond denotes the simulated results of the traditional acquisition. The asterisk denotes the simulated results of the joint acquisition with $M=4$. The circle denotes the simulated results of the joint acquisition with $M=8$. The cross denotes the simulated results of the joint acquisition with $M=12$. The square denotes the simulated results of the joint acquisition with $M=16$.

acquisition without satellite selection is affected seriously by the abnormal signals. In contrast, the JA with satellite selection is invulnerable to the abnormal signals.

\section{Conclusions}

The joint acquisition combining GNSS signals has been derived from a statistical point of view. The acquisition performance is detailedly analyzed in terms of the probabilities of false alarm and detection. A simplified implementing architecture is presented to reduce the computation load. In order to improve the robustness against the blocked satellites, the satellite selection strategy is proposed. Simulation results have validated the analytic expressions and demonstrated the benefit of the

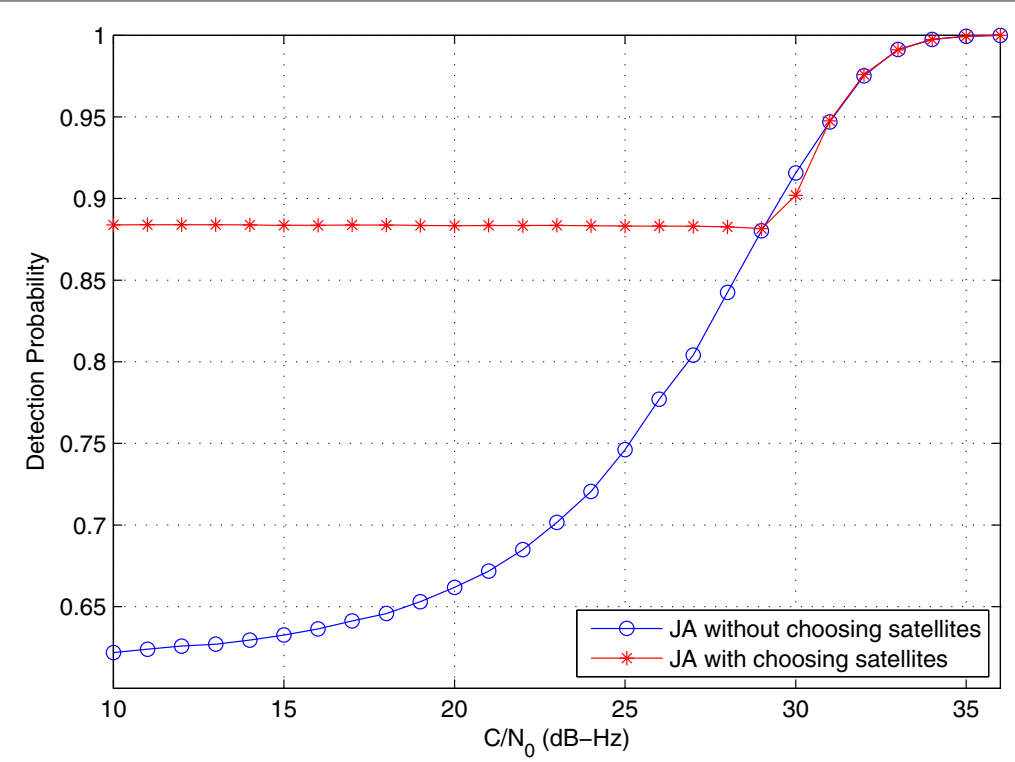

Figure 8 The detection probability with the satellite selection strategy. 
satellite selection strategy. The assisted joint acquisition also can be used in the reacquisition after lost tracking loop.

\section{Competing interests}

The authors declare that they have no competing interests.

\section{Author's information}

$H T$ is a Ph.D. candidate of the School of Electronic Science and Engineering at the National University of Defense Technology. His research interests include the GNSS signal acquisition and vector tracking.

\section{Acknowledgements}

This work has been supported by the China's Ministry of Education (NCET-08-0144).

\section{Received: 17 September 2013 Accepted: 28 April 2014}

Published: 19 May 2014

\section{References}

1. ED Kaplan, CJ Hegarty, Understanding GPS: Principles and Applications (Artech House, Boston, 2006)

2. R Watson, G Lachapelle, R Klukas, S Turunen, S Pietila, I Halivaara, Investigating GPS signals indoors with extreme high-sensitivity detection techniques. Navig. J. Inst. Navig. 52(4), 199-213 (2005)

3. N Ziedan, Global Navigation Satellite System (GNSS) Receivers for Weak Signals (Artech House, Boston, 2006)

4. SK Shanmugam, J Nielsen, G Lachapelle, Enhanced differential detection scheme for weak GPS signal acquisition, in 20th International Technical Meeting of the Satellite Division of The Institute of Navigation 2007 (ION GNSS 2007), (Fort Worth, 26-69 Sept 2007)

5. D Borio, D Akos, Noncoherent integrations for GNSS detection: analysis and comparisons. IEEE Trans. Aerosp. Electron. Syst. 45(1), 360-375 (2009). doi:10.1109/TAES.2009.4805285

6. D Borio, C Driscoll, G Lachapelle, Coherent, noncoherent, and differentially coherent combining techniques for acquisition of new composite GNSS signals. IEEE Trans. Aerosp. Electron. Syst. 45(3), 1227-1240 (2009). doi:10.1109/TAES.2009.5259196

7. JJ Spilker, The Global Positioning System: Theory and Applications, vol. 2. (AIAA, Washington, 1996)

8. T Pany, B Eissfeller, Use of a vector delay lock loop receiver for GNSS signal power analysis in bad signal conditions, in IEEE PLANS, Position Location and Navigation Symposium (San Diego, 25-27 April 2006)

9. M Lashley, DM Bevly, JY Hung, Performance analysis of vector tracking algorithms for weak GPS signals in high dynamics. IEEE J. Sel. Top. Sign. Proces. 3(4), 661-673 (2009). doi:10.1109/JSTSP.2009.2023341

10. P Closas, C Fernández-Prades, J Fernández-Rubio, Maximum likelihood estimation of position in GNSS. IEEE Signal Process. Lett. 14(5), 359-362 (2007). doi:10.1109/LSP.2006.888360

11. P Closas, C Fernández-Prades, J Fernández-Rubio, Cramér-Rao bound analysis of positioning approaches in GNSS receivers. IEEE Trans. Signal Process. 57(10), 3775-3786 (2009). doi:10.1109/TSP.2009.2025083

12. L Weill, A high performance code and carrier tracking architecture for ground-based mobile GNSS receivers, in 23rd International Technical Meeting of the Satellite Division of the Institute of Navigation 2010 (ION GNSS 2010), (Portland, 21-24 Sept 2010)

13. T Lin, JT Curran, C Driscoll, G Lachapelle, Implementation of a navigation domain GNSS signal tracking loop, (Portland, 19-21 September 2011)

14. P Axelrad, J Donna, M Mitchell, Signals from multiple channels and satellites, in 22nd International Technical Meeting of the Satellite Division of the Institute of Navigation 2009 (ION GNSS 2009) (Savannah, 22-25 Sept 2009)

15. BK Bradley, P Axelrad, J Donna, S Mohiuddin, Performance analysis of collective detection of weak GPS signals, in 23rd International Technical Meeting of the Satellite Division of the Institute of Navigation 2010 (ION GNSS 2010) (Portland, 21-24 Sept 2010)

16. P Axelrad, BK Bradley, J Donna, M Mitchell, S Mohiuddin, Collective detection and direct positioning using multiple GNSS satellites. Navig. J. Inst. Navig. 58(4), 305-321 (2011)
17. PK Sagiraju, GS Raju, D Akopian, Fast acquisition implementation for high sensitivity global positioning systems receivers based on joint and reduced space search. IET Radar Sonar Navig. 2(5), 376-387 (2008). doi:10.1049/iet-rsn:20070147

18. J Arribas, P Closas, C Fernández-Prades, Joint acquisition strategy of GNSS satellites for computational cost reduction, in 5th ESA Workshop on Satellite Navigation Technologies and European Workshop on GNSS Signals and Signal Processing (NAVITEC 2010) (Noordwijk, 8-10 Dec 2010)

19. LL Presti, $X$ Zhu, M Fantino, P Mulassano, GNSS signal acquisition in the presence of sign transition. IEEE J. Sel. Top. Sign. Proces. 3(4), 557-570 (2009). doi:10.1109/JSTSP.2009.2024592

20. SM Kay, Fundamentals of Statistical Signal Processing, Volumell: Detection Theory (Prentice Hall, New Jersey, 1993)

21. W Zhang, M Ghogho, Hypothesis testing analysis and unknown parameter estimation of GPS signal detection. J. Cent. South Univ Technol. Eng. Ed. 19(5), 1290-1301 (2012). doi:10.1007/s11771-012-1141-z

22. NL Knight, J Wang, C Rizos, Generalised measures of reliability for multiple outliers. J. Geod. 84(10), 625-635 (2009). doi:10.1007/s00190-010-0392-4

doi:10.1186/1687-6180-2014-73

Cite this article as: Tong et al: Joint acquisition algorithm with assisted information for weak GNSS signals. EURASIP Journal on Advances in Signal Processing 2014 2014:73.

\section{Submit your manuscript to a SpringerOpen ${ }^{\circ}$ journal and benefit from:}

- Convenient online submission

- Rigorous peer review

- Immediate publication on acceptance

- Open access: articles freely available online

- High visibility within the field

- Retaining the copyright to your article

Submit your next manuscript at $\boldsymbol{\wedge}$ springeropen.com 